\title{
LAUNCHING COSMIC-RAY-DRIVEN OUTFLOWS FROM THE MAGNETIZED INTERSTELLAR MEDIUM
}

\author{
Philipp Girichidis $^{1}$, Thorsten Naab ${ }^{1}$, Stefanie Walch ${ }^{2}$, Michą Hanasz ${ }^{3}$, Mordecai-Mark Mac Low ${ }^{4,5}$, \\ Jeremiah P. Ostriker ${ }^{6}$, Andrea Gatto ${ }^{1}$, Thomas Peters ${ }^{1}$, Richard WÜnsch ${ }^{7}$, Simon C. O. Glover ${ }^{5}$, \\ Ralf S. Klessen ${ }^{5}$, Paul C. Clark ${ }^{8}$, and Christian Baczynski ${ }^{5}$ \\ ${ }^{1}$ Max-Planck-Institut für Astrophysik, Karl-Schwarzschild-Str. 1, D-85741 Garching, Germany \\ ${ }^{2}$ Physikalisches Institut, Universität zu Köln, Zülpicher Str. 77, D-50937 Köln, Germany \\ ${ }^{3}$ Centre for Astronomy, Nicolaus Copernicus University, Faculty of Physics, Astronomy and Informatics, Grudziadzka 5, PL-87100 Toruń, Poland \\ ${ }^{4}$ Department of Astrophysics, American Museum of Natural History, 79th Street at Central Park West, New York, NY 10024, USA \\ ${ }^{5}$ Universität Heidelberg, Zentrum für Astronomie, Institut für Theoretische Astrophysik, Albert-Ueberle-Str. 2, D-69120 Heidelberg, Germany \\ ${ }^{6}$ Department of Astronomy, Columbia University, 1328 Pupin Hall, 550 West 120th Street, New York, NY 10027, USA \\ Astronomical Institute, Academy of Sciences of the Czech Republic, Bocni II 1401, 14131 Prague, Czech Republic \\ ${ }^{8}$ School of Physics \& Astronomy, Cardiff University, 5 The Parade, Cardiff CF24 3AA, Wales, UK \\ Received 2015 September 22; accepted 2015 December 10; published 2016 January 6
}

\section{ABSTRACT}

We present a hydrodynamical simulation of the turbulent, magnetized, supernova (SN)-driven interstellar medium (ISM) in a stratified box that dynamically couples the injection and evolution of cosmic rays (CRs) and a selfconsistent evolution of the chemical composition. CRs are treated as a relativistic fluid in the advection-diffusion approximation. The thermodynamic evolution of the gas is computed using a chemical network that follows the abundances of $\mathrm{H}^{+}, \mathrm{H}, \mathrm{H}_{2}, \mathrm{CO}, \mathrm{C}^{+}$, and free electrons and includes (self-)shielding of the gas and dust. We find that CRs perceptibly thicken the disk with the heights of $90 \%$ (70\%) enclosed mass reaching $\gtrsim 1.5 \mathrm{kpc}(\gtrsim 0.2 \mathrm{kpc})$. The simulations indicate that CRs alone can launch and sustain strong outflows of atomic and ionized gas with mass loading factors of order unity, even in solar neighborhood conditions and with a CR energy injection per SN of $10^{50} \mathrm{erg}, 10 \%$ of the fiducial thermal energy of an SN. The CR-driven outflows have moderate launching velocities close to the midplane $\left(\lesssim 100 \mathrm{~km} \mathrm{~s}^{-1}\right)$ and are denser $\left(\rho \sim 10^{-24}-10^{-26} \mathrm{~g} \mathrm{~cm}^{-3}\right)$, smoother, and colder than the (thermal) SN-driven winds. The simulations support the importance of CRs for setting the vertical structure of the disk as well as the driving of winds.

Key words: cosmic rays - diffusion - ISM: jets and outflows - ISM: structure - magnetohydrodynamics (MHD)

\section{INTRODUCTION}

Galactic outflows are important for the dynamical and chemical evolution of galaxies (e.g., Veilleux et al. 2005; Oppenheimer \& Davé 2006). They can enrich galactic halos with metals and regulate the baryonic angular momentum distribution in forming galaxies (e.g., Übler et al. 2014). Observations reveal that many actively star-forming galaxies in the early universe $(z \gtrsim 0.5)$ show wind signatures (e.g., Steidel et al. 2010; Newman et al. 2012; Rubin et al. 2014). In the local universe, strong winds are mainly associated with starbursts (e.g., M82 or NGC 253). Milky Way (MW)-type galaxies show galactic fountain features. Despite their importance, the driving mechanisms of galactic winds are not precisely known. Galaxy-scale sub-grid modes including supernova (SN) explosions, radiation pressure, and stellar winds only partially succeed in explaining observed galactic wind properties.

Recent numerical simulations of the galactic interstellar medium (ISM; Girichidis et al. 2015; Walch et al. 2015) have shown that the properties of SN-driven outflows are tightly connected to details of the ISM structure and the environmental densities of $\mathrm{SNe}$ as one of the main driving mechanisms. Most previous calculations of the SN-driven ISM recover the net global quantities like mass fractions and volume filling factors of the different thermal states of the gas close to the disk midplane (de Avillez \& Breitschwerdt 2005; Joung \& Mac Low 2006; Kim et al. 2013; Li et al. 2015). However, the vertical distribution of the gas above the disk as well as the temperature structure are not reproduced well by previous numerical models (Henley et al. 2010; Wood et al. 2010).
Cosmic rays (CRs) as a non-thermal component (see, e.g., Zweibel 2013; Grenier et al. 2015) might change the structures in the ISM even more. Their energy density is similar to the magnetic and thermal one (e.g., Cox 2005; Draine 2011 and references therein). As CRs do not cool as efficiently as the thermal gas, they provide a long-lived energy reservoir. Their transport through the medium can be described by a diffusion process relative to the gas, which allows CRs to populate large regions of the disk creating a steady pressure gradient. Both facts make CRs a fundamentally different dynamical driving mechanism in the ISM.

From theoretical considerations, a CR pressure gradient alone can generate outflows (Breitschwerdt et al. 1993; Everett et al. 2008; Dorfi \& Breitschwerdt 2012). This result is supported by recent galaxy-scale hydrodynamical simulations. Hanasz et al. (2013) neglect the thermal impact of SNe and show that CRs alone can launch fast winds from the magnetized ISM in high surface density disks $\left(\Sigma_{\text {gas }}=100 M_{\odot} \mathrm{pc}^{-2}\right)$. Booth et al. (2013) assume isotropic diffusion without the inclusion of magnetic fields and compare MW and Small Magellanic Cloud (SMC)-type galaxies, finding that CRs noticeably increase the mass loading factor $(\eta=\dot{M} / \mathrm{SFR})$ in SMC-like dwarf galaxies, but have a weaker impact in MW-type environments. With a similar implementation, Salem \& Bryan (2014) found stable outflows with $\eta=0.3$ but only for a high fraction of CR energy injection per SN. The main conclusion from these studies is that CRs can diffuse out of the dense regions of star formation and generate stable vertical pressure gradients. This helps to lift even dense and 
cold gas above the disk midplane. However, these models do not resolve the ISM structures and phases.

In this study, we present the first magnetohydrodynamical numerical simulations of stratified boxes that dynamically include CRs and follow the chemical evolution in the ISM. We aim for a more accurate description of the thermal and dynamical properties of a galactic disk with a focus on the vertical structure of the disk and the onset of outflows.

\section{NUMERICAL METHOD AND SIMULATION SETUP}

The simulations are performed with a modified version of the adaptive-mesh refinement code FLASH in version 4 (Fryxell et al. 2000; Dubey et al. 2008, 2013). We solve the magnetohydrodynamic (MHD) equations using the HLL3R method (Waagan 2009; Bouchut et al. 2010; Waagan et al. 2011), extended to a separate monoenergetic CR fluid similar to Yang et al. (2012). Tests of this implementation are presented in Girichidis et al. (2014).

The combined system of equations that we solve numerically is

$$
\begin{gathered}
\frac{\partial \rho}{\partial t}+\nabla \cdot(\rho \boldsymbol{v})=0 \\
\frac{\partial \rho \boldsymbol{v}}{\partial t}+\nabla \cdot\left(\rho \boldsymbol{v} \boldsymbol{v}^{\mathrm{T}}-\frac{\boldsymbol{B} \boldsymbol{B}^{\mathrm{T}}}{4 \pi}\right)+\nabla p_{\mathrm{tot}}=\rho \boldsymbol{g} \\
\frac{\partial e}{\partial t}+\nabla \cdot\left[\left(e+p_{\mathrm{tot}}\right) \boldsymbol{v}-\frac{\boldsymbol{B}(\boldsymbol{B} \cdot \boldsymbol{v})}{4 \pi}\right] \\
=\rho \boldsymbol{v} \cdot \boldsymbol{g}+\nabla \cdot \mathrm{K} \nabla e_{\mathrm{CR}}+\dot{u}_{\mathrm{chem}}+\dot{u}_{\mathrm{inj}} \\
\frac{\partial \boldsymbol{B}}{\partial t}-\nabla \times(\boldsymbol{v} \times \boldsymbol{B})=0 \\
\frac{\partial e_{\mathrm{CR}}}{\partial t}+\nabla \cdot\left(e_{\mathrm{CR}} \boldsymbol{v}\right) \\
=-p_{\mathrm{CR}} \nabla \cdot \boldsymbol{v}+\nabla \cdot\left(\mathrm{K} \nabla e_{\mathrm{CR}}\right)+Q_{\mathrm{CR}} .
\end{gathered}
$$

Here, $\rho$ is the mass density, $\boldsymbol{v}$ is the velocity, and $\boldsymbol{B}$ is the magnetic field. The total energy density,

$$
e=\rho v^{2} / 2+e_{\mathrm{th}}+e_{\mathrm{CR}}+B^{2} / 8 \pi,
$$

includes kinetic, thermal, $\mathrm{CR}$, and magnetic contributions. We evolve the $\mathrm{CR}$ energy density, $e_{\mathrm{CR}}$, separately. The total pressure is

$$
\begin{gathered}
p_{\mathrm{tot}}=p_{\mathrm{th}}+p_{\mathrm{CR}}+p_{\mathrm{mag}} \\
=(\gamma-1) e_{\mathrm{th}}+\left(\gamma_{\mathrm{CR}}-1\right) e_{\mathrm{CR}}+B^{2} / 8 \pi .
\end{gathered}
$$

The closure relation for the system, the equation of state, combines the different contributions from CR and thermal pressure in an effective adiabatic index, $\gamma_{\mathrm{eff}}$,

$$
\gamma_{\mathrm{eff}}=\frac{\gamma p_{\mathrm{th}}+\gamma_{\mathrm{CR}} p_{\mathrm{CR}}}{p_{\mathrm{th}}+p_{\mathrm{CR}}},
$$

where we set $\gamma=5 / 3$ and $\gamma_{\mathrm{CR}}=4 / 3$ for gas and CRs, respectively. For the $\mathrm{CR}$ diffusion tensor, $\mathrm{K}$, we assume a value of $10^{28} \mathrm{~cm}^{2} \mathrm{~s}^{-1}$ parallel and $10^{26} \mathrm{~cm}^{2} \mathrm{~s}^{-1}$ perpendicular to the magnetic field lines (e.g., Strong et al. 2007; Nava \& Gabici 2013).

An exact treatment of CR propagation and the coupling to the gas would include the generation of Alfvén waves due to CR streaming and wave damping due to ion-neutral collisions and nonlinear Landau damping including anisotropic particle distribution functions (e.g., Kulsrud \& Pearce 1969; Ptuskin et al. 1997; Breitschwerdt et al. 2002; Dorfi \& Breitschwerdt 2012; Uhlig et al. 2012; Wiener et al. 2013). However, the detailed coupling of CRs to the gas-in particular, the weakly ionized gas - is an open question and beyond the scope of this Letter. For our model we assume that CRs are scattered sufficiently by small-scale background turbulence, which we do not resolve. We therefore adopt the simplified transport equation following Schlickeiser \& Lerche (1985; see also Yang et al. 2012; Hanasz et al. 2013), which is derived from an isotropic particle distribution function.

Radiative and chemical heating and cooling, $\dot{u}_{\text {chem, are }}$ computed using a chemical network that follows the abundances of $\mathrm{H}^{+}, \mathrm{H}, \mathrm{H}_{2}, \mathrm{C}^{+}, \mathrm{CO}$, and free electrons (see Glover \& Clark 2012 and references therein). We assume a constant $\mathrm{CR}$ ionization rate $\left(3 \times 10^{-17} \mathrm{~s}\right)$. We follow (self-) shielding of the molecular gas as described in Clark et al. (2012). Photoionization is neglected. The thermal and CR energy injected by the $\mathrm{SNe}$ are included in $\dot{u}_{\text {inj }}$ and $Q_{\mathrm{CR}}$. The external gravitational acceleration, $\boldsymbol{g}$, is taken from Kuijken \& Gilmore (1989).

The stratified disk is initially atomic, at rest, and in pressure equilibrium with a scale height of $60 \mathrm{pc}$ and a gas surface density of $10 M_{\odot} \mathrm{pc}^{-2}$ (see Walch et al. 2015 for further details) in a box of $2 \times 2 \times \pm 20 \mathrm{kpc}$. For $|z|<2.5 \mathrm{kpc}$, we adopt a cell size of $15.6 \mathrm{pc}$, which corresponds to an effective numerical resolution of $128 \times 128 \times 2560$ cells. For $|z|>2.5 \mathrm{kpc}$, the adaptive-mesh refinement can coarsen the resolution. Motivated by the Kennicutt-Schmidt relation (Kennicutt 1998), we explode $\mathrm{SNe}$ at a fixed rate of $60 \mathrm{Myr}^{-1} \mathrm{kpc}^{-2}$ assuming one massive star per $100 M_{\odot}$. Similar to de Avillez \& Breitschwerdt (2004) or Joung \& Mac Low (2006), we assign $20 \%$ of the explosions to SN Ia and $80 \%$ to SN II with scale heights of $325 \mathrm{pc}$ and $50 \mathrm{pc}$, respectively (Tammann et al. 1994). However, estimates of the displacement of runaway OB stars suggests that the effective scale height of SNe II might be larger (Li et al. 2015).

We separately vary the thermal energy injection per $\mathrm{SN}$, $E_{\mathrm{th}}=f_{\mathrm{th}} E_{\mathrm{SN}}$, and the $\mathrm{CR}$ energy injection, $E_{\mathrm{CR}}=f_{\mathrm{CR}} E_{\mathrm{SN}}$, with $E_{\mathrm{SN}}=10^{51} \mathrm{erg}$. We discuss three different simulations: purely thermal $\mathrm{SNe}\left(f_{\mathrm{th}}=1.0, f_{\mathrm{CR}}=0.0, \mathrm{TH} 1-\mathrm{CR} 0\right)$, purely CR SNe $\left(f_{\text {th }}=0.0, f_{\mathrm{CR}}=0.1\right.$, TH0-CR 1$)$, and the physically most realistic setup with both energy injections $\left(f_{\mathrm{th}}=1.0, f_{\mathrm{CR}}=0.1, \mathrm{TH} 1-\mathrm{CR} 1\right)$.

\section{MORPHOLOGICAL EVOLUTION}

In all simulations, the $\mathrm{SNe}$ induce the formation of filaments, clumps, and voids. The presence of CRs leads to a smoother morphological appearance. An overview of the disk structure after $250 \mathrm{Myr}$ is depicted in Figure 1 with cuts through the center of the box for the density edge-on (top) and face-on (middle) and the CR energy density (bottom). From left to right, we present $\mathrm{TH} 1-\mathrm{CR} 0, \mathrm{TH} 0-\mathrm{CR} 1$, and $\mathrm{TH} 1-\mathrm{CR} 1$. Both runs with CRs quickly push gas away from the midplane and form an extended atmosphere, whereas in the simulation with only thermal SN feedback, the disk remains compact without a noticeable extended layer of gas above the disk. The CR energy density shows relatively small spatial variations of about one order of magnitude. High CR energy peaks correspond to recent $\mathrm{SNe}$ and disappear quickly due to diffusion.

In Figure 2, we show the vertical profile of the density for all simulations at $t=250 \mathrm{Myr}$. The arrows indicate the heights of 

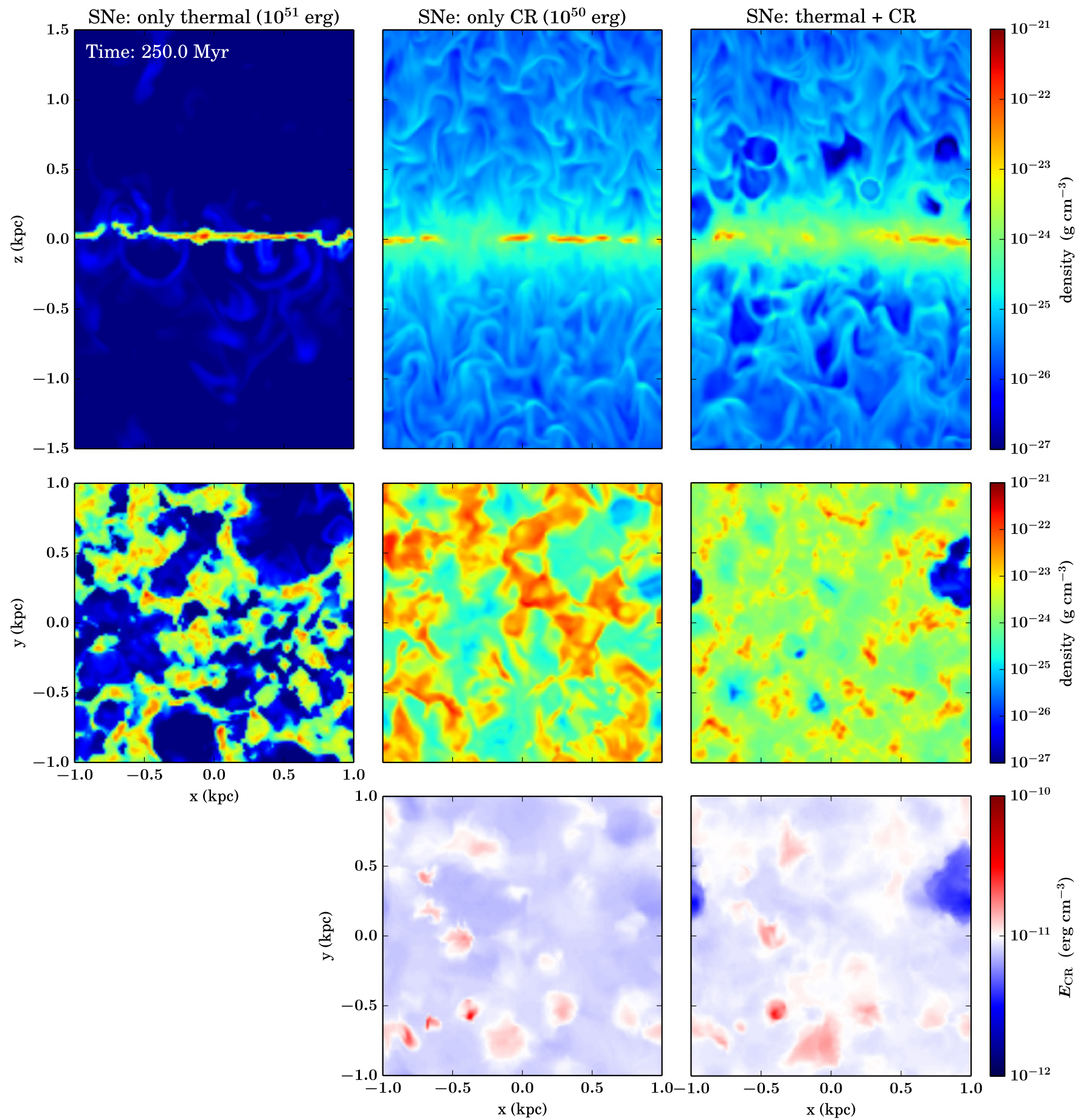

Figure 1. Structure of the disk after $250 \mathrm{Myr}$ of evolution. The left panel shows the run without CRs, the middle panel the run with purely CR feedback, and the right panel the simulation with both feedback energies. In the top and middle row, we plot the density edge-on and face-on in cuts through the center of the box. The bottom row depicts the CR energy density in the midplane. The disk in the run including CRs is significantly thicker compared to the purely thermal run. The thermal energy input of the $\mathrm{SNe}$ is primarily important in the denser part of the disk. CRs show their main impact in generating an extended atmosphere. The CR energy density is smooth in the midplane. Local variations are due to recent SNe and the resulting CR injection. Those variations disappear after less than a Myr due to diffusion.

90\% enclosed mass. The inclusion of CRs increases $H_{90 \%}$ from $\sim 30$ to $\sim 1500$ pc with similar values for both runs including CRs. The additional thermal energy injection from $\mathrm{SNe}$ in run TH1-CR1 changes the profile up to a height of $\sim 300 \mathrm{pc}$. At larger altitudes, the profiles for THO-CR1 and $\mathrm{TH} 1-\mathrm{CR} 1$ are basically indistinguishable. For comparison, we include the vertical density profile of the MW (Dickey \& Lockman 1990) showing reasonable agreement with both CR runs.
The time evolution of the disk thickness is shown in Figure 3 including 70\% (solid line) and 90\% (dashed line) of enclosed mass. Run TH1-CRO shows little variation of the disk over the entire simulation time. For the other runs, the CR energy density can push a significant fraction of the gas to heights of up to $\sim 400 \mathrm{pc}$ in the beginning of the simulation before the initially ordered magnetic field becomes tangled (Parker instability) and the CR energy quickly diffuses along the 


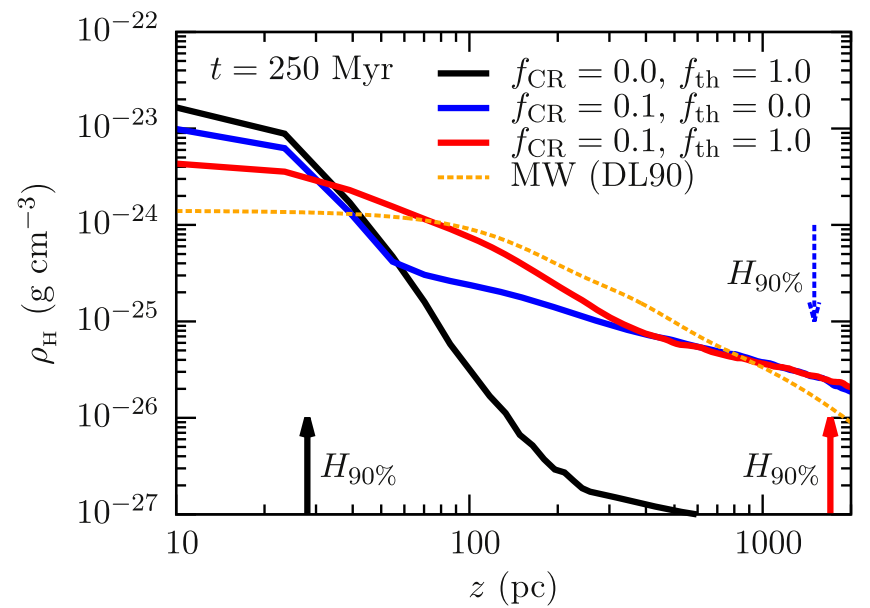

Figure 2. Vertical profiles of the total gas density for all simulations. The arrows indicate the height of $90 \%$ enclosed mass. A fit to the observed density profile of the solar neighborhood (Dickey \& Lockman 1990) are shown in yellow. Thermal energy injection alone leads to a compact atomic gas distribution. Including CR feedback results in very extended distributions, which are much closer to the observed extent of the gas. The profiles indicate that CRs have their main impact at larger altitudes.

opened up field lines. Opening magnetic field lines enable the reduction of CR pressure via the escape of excessive $\mathrm{CR}$ gas initially trapped in the horizontal magnetic field. The bulk of the disk thickens slowly over time $\left(H_{70 \%}\right)$, whereas the envelope expands quickly $\left(H_{90 \%}\right)$.

\section{OUTFLOWS}

The properties of the outflows are plotted in Figure 4, with the total outflow rate (top), the ratio of atomic to ionized hydrogen (middle), and the temperature (bottom), averaged at $\pm 1 \mathrm{kpc}$. Included is all the gas with velocities pointing away from the midplane. All simulations drive outflows at a roughly constant rate after $\sim 50 \mathrm{Myr}$. Run $\mathrm{TH} 1-\mathrm{CRO}$ reaches a mass loading factor, $\eta=\dot{M} / \mathrm{SFR} \sim 0.1-0.2$ at $1 \mathrm{kpc}$ above the midplane. Including $\mathrm{CRs}$ increases $\eta$ to order unity. This indicates that in our setups the main driver for outflows is CRs rather than the thermal contribution from the $\mathrm{SNe}$. The chemical composition, however, is affected by the thermal contribution. The outflowing gas in the simulation with purely thermal SN feedback is very hot $\left(10^{7} \mathrm{~K}\right.$; bottom panel of Figure 4) and composed entirely of ionized hydrogen $\mathrm{H}^{+}$(not visible). In the other two cases with CRs, the outflowing gas is warm $\left(\sim 10^{4} \mathrm{~K}\right)$ and mainly composed of atomic hydrogen with $\dot{M}_{\mathrm{H}} / \dot{M}_{\mathrm{H}+} \sim 5-10$. The inclusion of photoionization feedback is likely to decrease this value, but will not significantly alter the temperature of the outflowing gas or the very low volumefilling fractions of the hot gas. As soon as the CR pressure gradient has built up, significant fractions of neutral and warm gas can be accelerated away from the disk midplane (see, e.g., Salem \& Bryan 2014).

The connection between density and outflow velocity is depicted in Figure 5. For $\mathrm{TH} 1-\mathrm{CRO}$ (top panel), the $\mathrm{SNe}$ explode partially in regions of very low density, can thus expand to large radii, and push gas out of the midplane at very high velocities of up to $\sim 500 \mathrm{~km} \mathrm{~s}^{-1}$ at very low densities below $\rho \sim 10^{-26} \mathrm{~g} \mathrm{~cm}^{-3}$. If CRs are included, gas is slowly transported away from the dense regions in the midplane to form an extended atmosphere, whose flow of gas becomes perceptibly denser and overall slower compared to TH1-CRO. At the low heights investigated here, the gas does not reach escape velocity. Again, this is because the CRs quickly diffuse throughout the volume and build up a stable, slowly varying energy (pressure) gradient along the $z$-direction that slowly but continuously accelerates the gas, in contrast to the short-lived locally acting thermal energy input. This underlines the fundamental difference of galactic winds driven by $\mathrm{SNe}$ and CRs. The comparison between THO-CR1 (middle) and the combined energy input (TH1-CR1) shows only little impact on the bulk density of the outflow $\left(\rho \sim 10^{-26}-10^{-24} \mathrm{~g} \mathrm{~cm}^{-3}\right)$. The contribution of the thermal component in the $\mathrm{SN}$ driving increases the peak velocities slightly from $\sim 50$ to $\sim 100 \mathrm{~km} \mathrm{~s}^{-1}$. However, the dense atmosphere around the midplane, in which the SNe explode, reduces their net impact on the velocities compared to $\mathrm{TH} 1-\mathrm{CRO}$ (see also Girichidis et al. 2015). For observational signatures in the soft X-ray emission, see Peters et al. (2015).

\section{DISCUSSION}

We find that CRs are able to perceptibly increase the thickness of the disk and launch and sustain strong outflows with mass loading factors of order unity. For the simulations presented here, the CR-driven winds are slower (50-100 $\mathrm{km} \mathrm{s}^{-1}$ ) and denser than the (pure thermally) SNdriven winds. This is qualitatively consistent with the results by Booth et al. (2013). Salem \& Bryan (2014) report mass loading factors below unity for their fiducial model with a total CR fraction per SN of $30 \%$ rather than our $10 \%$. The simulations of Hanasz et al. (2013) also show mass loading factors above unity but for massive galactic disks with high $\mathrm{SN}$ rates. We note, however, that an important parameter of the model is the exact value of the assumed CR diffusion coefficient. Its value controls both the terminal wind speed and mass-loss rate (Dorfi $\&$ Breitschwerdt 2012). For small values of K, mass-loss rates are higher at lower speeds, whereas large diffusion coefficients result in lower mass loading factors but higher velocities. We found a similar behavior in tests with varying diffusion tensor for the setup described here. Full details will be provided in a follow-up paper.

Previous models of stratified disks have shown that magnetic fields help to increase the thickness of the disk (Hill et al. 2012), but that they alone are not strong enough to reach observed scale heights. The combined MHD-CR simulation presented here seems a promising solution to this problem.

We also note that the impact of purely thermal SNe strongly depends on the positioning of the $\mathrm{SNe}$ and therefore might be resolution dependent. In particular, the $\mathrm{SNe}$ that explode in density peaks have a weaker net impact (Gatto et al. 2015; Girichidis et al. 2015; Walch et al. 2015), and thus the outflow rates in the purely thermal run, $\mathrm{TH} 1-\mathrm{CR} 0$, might be lower limits. This weak SN efficiency might also be responsible for small fractions of hot gas in the CR runs. However, we find that the inclusion of CRs results in outflows that are compatible with the observations. This suggests that CRs will always play an important role in the matter cycle of galaxies.

\section{CONCLUSIONS}

We present the first ISM simulations that dynamically couple CRs with the chemodynamical evolution of the magnetized ISM. The CRs are included in an advection-diffusion approach 

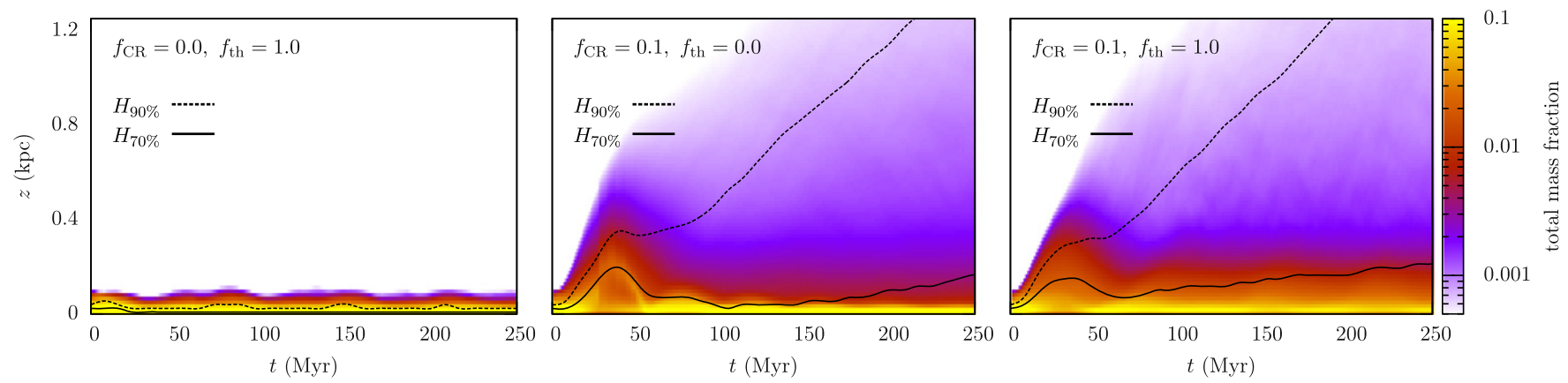

Figure 3. Time evolution of the vertical gas distribution. Color coded is the total gas fraction. Overplotted are the heights of $90 \%\left(H_{90 \%}\right.$, dashed line) and $70 \%\left(H_{70 \%}\right.$, solid line) enclosed total mass. In the purely thermal run (left) the disk does not show variations over time. Including CRs leads to a slow expansion of bulk of the disk $\left(H_{70 \%}\right)$ and a fast expansion of the envelope $\left(H_{90 \%}\right)$.
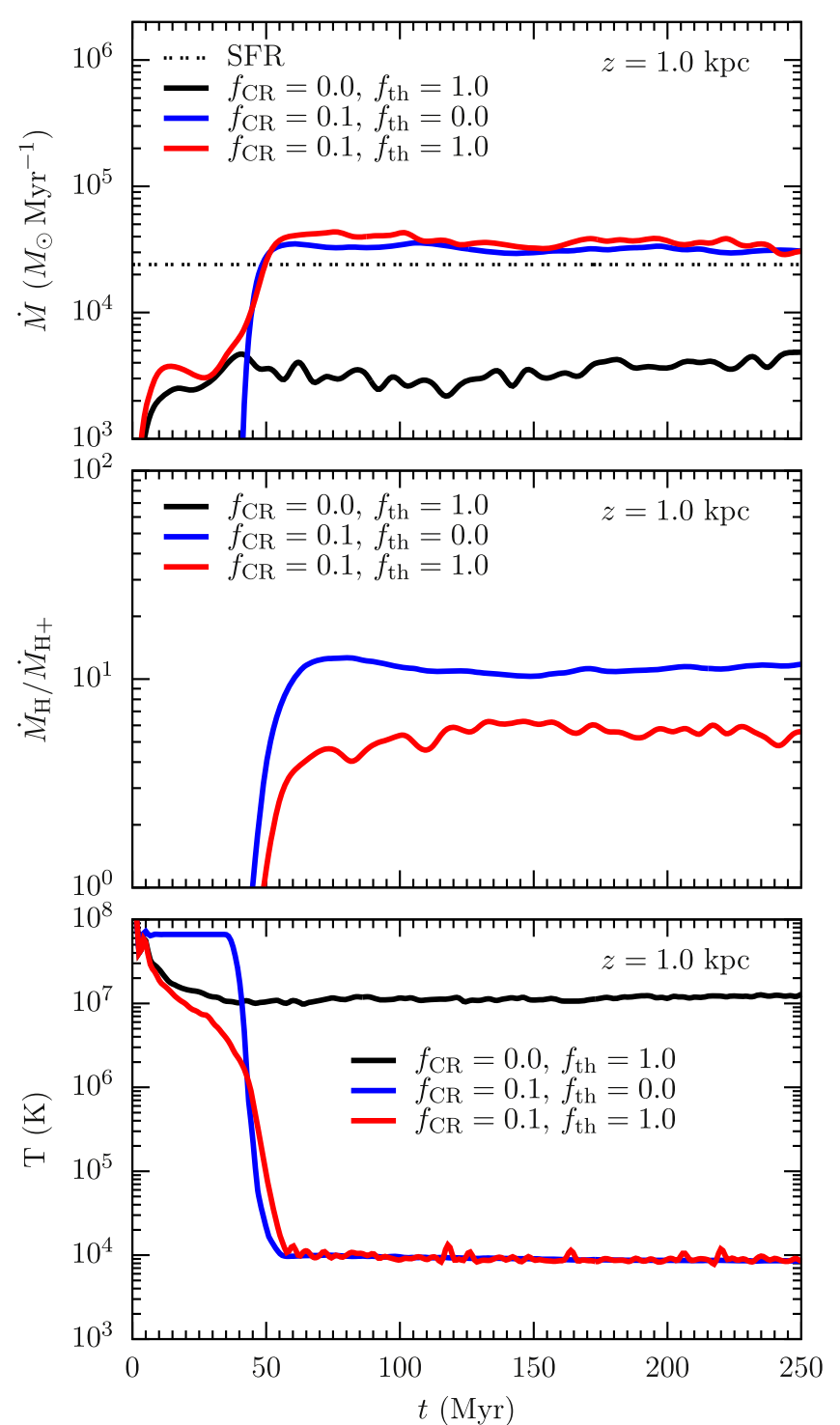

Figure 4. Outflow rate (top), the ratio of atomic to ionized hydrogen (middle), and the temperature (bottom) at $z= \pm 1 \mathrm{kpc}$. All simulations show outflows. The purely thermal $\mathrm{SNe}$ have outflow rates lower than the star formation rate. Including CRs, the mass loading factor increases to unity. The outflows driven by purely thermal SNe are hot $\left(\sim 10^{7} \mathrm{~K}\right)$ and consist of ionized hydrogen. The CR-driven counterparts are colder $\left(10^{4} \mathrm{~K}\right)$ and mainly composed of atomic hydrogen.

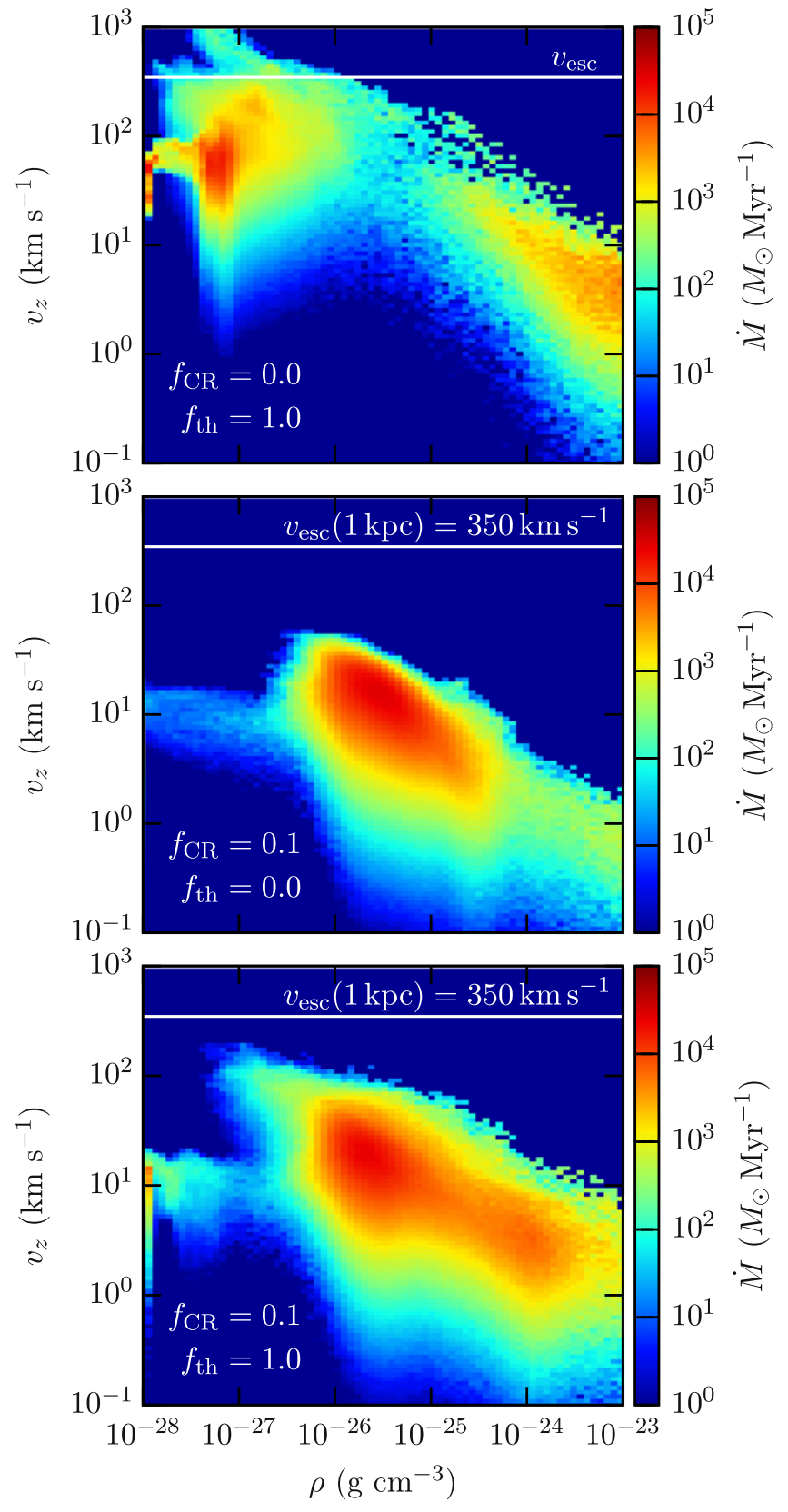

Figure 5. Histograms of the outflows $\left(v_{z} z>0\right)$ as a function of density and velocity. Purely thermal SN feedback (top) leads to fast low-density outflows. Including CRs (middle and bottom) the transported gas is two orders of magnitude denser and a factor of a few slower with broader distributions. 
with an anisotropic diffusion tensor. We follow the formation of density structures, the vertical distribution of gas in a galactic disk, and the launching of outflows in three different runs varying the $\mathrm{SN}$ feedback between purely thermal, purely $\mathrm{CR}$, and combined thermal and CR feedback. Our conclusions can be summarized as follows:

1. Including CRs thickens the galactic disk. The height of $90 \%$ enclosed total mass is found to be $\sim 1.5 \mathrm{kpc}$ in the case of $10 \% \mathrm{CR}$ energy injection per SN after $250 \mathrm{Myr}$ and to increase continuously. Comparison with the vertical density distribution in the MW indicates good agreement.

2. We find that CRs quickly lead to the formation of a warm and neutral galactic atmosphere providing a mass reservoir for galactic winds and outflows. Whereas the thermal contribution of the SNe mainly shapes the disk close to the midplane, the additional CR energy shows the strongest impact above the disk and in the halo.

3. All simulations drive gas out of the midplane with little variation over time. For purely thermal SN feedback, the outflows are hot and composed of mainly ionized hydrogen with rates below the star formation rate. They are fast (up to $\sim$ a few $100 \mathrm{~km} \mathrm{~s}^{-1}$ ) with low densities $\left(\rho \lesssim 10^{-27} \mathrm{~g} \mathrm{~cm}^{-3}\right)$. CRs alone can drive outflows with mass loading factors of order unity, which are warm $\left(10^{4} \mathrm{~K}\right)$ and mainly composed of atomic hydrogen. They are a factor of a few slower $\left(\sim 10-50 \mathrm{~km} \mathrm{~s}^{-1}\right)$ and $1-2$ orders of magnitude denser $\left(\rho \sim 10^{-26}-10^{-25} \mathrm{~g} \mathrm{~cm}^{-3}\right)$ compare to their thermally driven counterparts.

We thank the referee for valuable comments and questions. P.G., S.W., T.N., T.P., A.G., S.C.O.G., R.S.K., and C.B. acknowledge support from the DFG Priority Program 1573 Physics of the Interstellar Medium. S.W. acknowledges the support of the Bonn-Cologne Graduate School, which is funded through the Excellence Initiative. T.N. acknowledges support from the DFG cluster of excellence Origin and Structure of the Universe. R.W. acknowledges support by the Czech Science Foundation grant 209/12/1795 and by the project RVO:67985815 of the Academy of Sciences of the Czech Republic. R.S.K., S.C.O.G., and C.B. thank the DFG for funding via the SFB 881 The Milky Way System (subprojects B1, B2, and B8). R.S.K. furthermore acknowledges support from the European Research Council under the European Community's Seventh Framework Programme (FP7/ 2007-2013) via the ERC Advanced Grant STARLIGHT (project number 339177). M.-M.M.L. was partly supported by NSF grant AST11-09395 and the Alexander von Humboldt Foundation. M.H. acknowledges support from Polish NCN grant N203 511038. The authors thank the Max Planck Computing and Data Facility (MPCDF) for computing time and data storage. The software used in this work was developed in part by the DOE NNSA ASC- and DOE Office of Science
ASCR-supported Flash Center for Computational Science at the University of Chicago.

\section{REFERENCES}

Booth, C. M., Agertz, O., Kravtsov, A. V., \& Gnedin, N. Y. 2013, ApJL, 777, L16

Bouchut, F., Klingenberg, C., \& Waagan, K. 2010, NuMat, 115, 647

Breitschwerdt, D., Dogiel, V. A., \& Völk, H. J. 2002, A\&A, 385, 216

Breitschwerdt, D., McKenzie, J. F., \& Voelk, H. J. 1993, A\&A, 269, 54

Clark, P. C., Glover, S. C. O., \& Klessen, R. S. 2012, MNRAS, 420, 745

Cox, D. P. 2005, ARA\&A, 43, 337

de Avillez, M. A., \& Breitschwerdt, D. 2004, A\&A, 425, 899

de Avillez, M. A., \& Breitschwerdt, D. 2005, A\&A, 436, 585

Dickey, J. M., \& Lockman, F. J. 1990, ARA\&A, 28, 215

Dorfi, E. A., \& Breitschwerdt, D. 2012, A\&A, 540, A77

Draine, B. T. 2011, Physics of the Interstellar and Intergalactic Medium (Princeton, NJ: Princeton Univ. Press)

Dubey, A., Fisher, R., Graziani, C., et al. 2008, in ASP Conf. Ser. 385 , Numerical Modeling of Space Plasma Flows: Astronum 2007, ed. N. V. Pogorelov, E. Audit, \& G. P. Zank (San Francisco, CA: ASP), 145

Dubey, A., et al. 2013, in SE-CSE 2013: The 2013 Int. Workshop on Software Engineering for Computational Science and Engineering, 1

Everett, J. E., Zweibel, E. G., Benjamin, R. A., et al. 2008, ApJ, 674, 258

Fryxell, B., Olson, K., Ricker, P., et al. 2000, ApJS, 131, 273

Gatto, A., Walch, S., Mac Low, M.-M., et al. 2015, MNRAS, 449, 1057

Girichidis, P., Naab, T., Walch, S., \& Hanasz, M. 2014, arXiv:1406.4861

Girichidis, P., Walch, S., Naab, T., et al. 2015, arXiv:1508.06646

Glover, S. C. O., \& Clark, P. C. 2012, MNRAS, 421, 116

Grenier, I. A., Black, J. H., \& Strong, A. W. 2015, ARA\&A, 53, 199

Hanasz, M., Lesch, H., Naab, T., et al. 2013, ApJL, 777, L38

Henley, D. B., Shelton, R. L., Kwak, K., Joung, M. R., \& Mac Low, M.-M. 2010, ApJ, 723, 935

Hill, A. S., Joung, M. R., Mac Low, M.-M., et al. 2012, ApJ, 750, 104

Joung, M. K. R., \& Mac Low, M.-M. 2006, ApJ, 653, 1266

Kennicutt, R. C., Jr. 1998, ApJ, 498, 541

Kim, C.-G., Ostriker, E. C., \& Kim, W.-T. 2013, ApJ, 776, 1

Kuijken, K., \& Gilmore, G. 1989, MNRAS, 239, 605

Kulsrud, R., \& Pearce, W. P. 1969, ApJ, 156, 445

Li, M., Ostriker, J. P., Cen, R., Bryan, G. L., \& Naab, T. 2015, ApJ, 814, 4

Nava, L., \& Gabici, S. 2013, MNRAS, 429, 1643

Newman, S. F., Genzel, R., Förster-Schreiber, N. M., et al. 2012, ApJ, 761, 43

Oppenheimer, B. D., \& Davé, R. 2006, MNRAS, 373, 1265

Peters, T., Girichidis, P., Gatto, A., et al. 2015, ApJL, 813, 27

Ptuskin, V. S., Voelk, H. J., Zirakashvili, V. N., \& Breitschwerdt, D. 1997, A\&A, 321, 434

Rubin, K. H. R., Prochaska, J. X., Koo, D. C., et al. 2014, ApJ, 794, 156

Salem, M., \& Bryan, G. L. 2014, MNRAS, 437, 3312

Schlickeiser, R., \& Lerche, I. 1985, A\&A, 151, 151

Steidel, C. C., Erb, D. K., Shapley, A. E., et al. 2010, ApJ, 717, 289

Strong, A. W., Moskalenko, I. V., \& Ptuskin, V. S. 2007, ARNPS, 57, 285

Tammann, G. A., Loeffler, W., \& Schroeder, A. 1994, ApJS, 92, 487

Übler, H., Naab, T., Oser, L., et al. 2014, MNRAS, 443, 2092

Uhlig, M., Pfrommer, C., Sharma, M., et al. 2012, MNRAS, 423, 2374

Veilleux, S., Cecil, G., \& Bland-Hawthorn, J. 2005, ARA\&A, 43, 769

Waagan, K. 2009, JCoPh, 228, 8609

Waagan, K., Federrath, C., \& Klingenberg, C. 2011, JCoPh, 230, 3331

Walch, S., Girichidis, P., Naab, T., et al. 2015, MNRAS, 454, 238

Wiener, J., Zweibel, E. G., \& Oh, S. P. 2013, ApJ, 767, 87

Wood, K., Hill, A. S., Joung, M. R., et al. 2010, ApJ, 721, 1397

Yang, H.-Y. K., Ruszkowski, M., Ricker, P. M., Zweibel, E., \& Lee, D. 2012, ApJ, 761, 185

Zweibel, E. G. 2013, PhPl, 20, 055501 